\title{
EVOLUTION OF VERY LUMINOUS INFRARED GALAXIES
}

\author{
H. WU AND Z.L. ZOU \\ Beijing Astronomical Observatory, P.R. China \\ X.Y. XIA \\ Dept. of Physics, Tianjin Normal University, P.R.China \\ AND \\ Z. G. DENG \\ Dept. of Physics, Graduate School, USTC, P.R.China
}

We have completed spectroscopic observations (Wu et al. 1997a) of a sample of 73 very luminous infrared galaxies $\left(\log \left(L_{\mathrm{IR}} / L_{\odot}\right) \geq 11.5 ; H_{0}=50 \mathrm{~km}\right.$ $\mathrm{s}^{-1} \mathrm{Mpc}^{-1}$ ) from the 2-Jy catalogue (Strauss et al. 1992) using the $2.16 \mathrm{~m}$ telescope at the Beijing Astronomical Observatory. Spectral and interacting classifications are performed for the sample (Wu et al. 1997b). These statistical results provide strong evidence for the idea that interactions trigger nuclear activity and enhance the infrared luminosity. With the decrease of nuclear separation, relative velocity and specific angular momentum decrease rapidly, while on the contrary, both infrared luminosity and $\mathrm{H} \alpha$ equivalent width increase. Dynamical friction plays an important role even when two galaxies have large separation. This provides a favorable condition for strong star formation. We construct a simple merger sequence, from interaction class 1 to 4 , to class 5 and 6 and then to the class 0 regarded as being in the stage of advanced merger. Along this sequence, spectral types change from HII-like to AGN-like. Considering the strong correlation of very luminous infrared galaxies in spectral classification schemes, it is reliable that infrared luminous galaxies evolve from HII-like galaxies to AGN-like galaxies. The different properties of infrared luminous Seyfert 1s and optically selected Seyfert 1s suggest that infrared luminous galaxies could evolve into optical Seyfert 1s in the last stage.

\section{References}

Strauss, M.A., et al. 1992, $A p J S, \mathbf{8 3}, 29$

Wu, H., Zou, Z.L., Xia, X.Y., \& Deng, Z.G. 1997a, $A \& A S$, accepted

Wu, H., Zou, Z.L., Xia, X.Y., \& Deng, Z.G. 1997b, in preparation 